\title{
The Role of Homocysteine and Other Clinical Laboratory Markers in Assessing Cardiovascular Risk in Patients on Hemodialysis
}

Irena Ivanova Gencheva-Angelova, Adelaida Lazarova Ruseva and Pavlina Dimitrova Yordanova-Laleva UMHAT, Medical University, Pleven 5800, Bulgaria

\begin{abstract}
Cardiovascular diseases constitute approximately 50\% of deaths among dialysis patients in the USA and Europe. The increase in traditional and nontraditional cardiovascular risk factors in determining the high mortality of patients with end-stage renal disease (ESRD) is complicated due to the high frequency of risk factors in these patients. Some laboratory markers like homocysteine, albumin, cholesterol, triglycerides, LDL-cholesterol, and creatinine could be efficient in marking the risk of cardiovascular disease in these patients. We use Roche assay tests, based on routinely principles to determine this laboratory parameters used in the clinical laboratory. All laboratory parameters we measured on a biochemistry auto analyser Cobas Integra 400 at the clinical laboratory of University Hospital-Pleven. Using a statistical program a research was done on the quantitative characteristics and prognostic capabilities of homocysteine and other biochemical parameters. We determined the diagnostic specificity and sensitivity of our lab performance against vascular disease (heart attack or stroke) by ROC curves. For each of the observed values of biochemical parameters we calculated the diagnostic sensitivity and specificity. The threshold values for which the parameters have the highest sensitivity and specificity have been concluded. Summary of diagnostic value of parameters to judge the coefficient AUC - area under the curve, for cholesterol, LDL, triglycerides, albumin, it was a significant $(P<0.05)$. Homocysteine and the rest of the studied by us laboratory parameters can be regarded as laboratory markers of choice for assessing the risk of heart attack or stroke in patients on dialysis.
\end{abstract}

Key words: Homocysteine, albumin, end-stage renal disease, cardiovascular risk factors.

\section{Introduction}

Cardiovascular diseases form approximately $50 \%$ of deaths among dialysis patients in the USA and Europe [1]. The increase in traditional and nontraditional cardiovascular risk factors in determining the high mortality of patients with end-stage renal disease (ESRD) is complicated due to the high frequency of risk factors in these patients [2]. Hyperhomocysteinemia is seen as a cardiovascular risk factor in patients with chronic renal damage [3] and the issue is analyzed in several studies [4-12]. In relation to this, the observation that plasma homocysteine level (Hcy) is independently connected to the aortic injury [13], and left ventricular hypertrophy [14] are of particular interest. However,

Corresponding author: Irena Ivanova Gencheva-Angelova, $\mathrm{MD}$, research field: clinical laboratory. with the exception of research Bostom et al. [15] and Moustapha et al. [16] in a total of 240 patients there is a lack of connection between hyperhomocysteinemia and cardiovascular mortality in hemodialysis patients. The issue is mainly important because a recent study probed that the reduced concentration of plasma homocysteine, but not hyperhomocysteinemia, predicts reducing of the risk of vascular events [17].

The elevated Hcy shows atherogenic and prothrombotic properties and histopathologic features of Hcy induced vascular damage. It is included thickening of the intimal disruption elastic layer vascular smooth muscle hypertrophy, marked accumulation of platelets and the formation of platelet-enriched blood clots. The role of homocysteine in coronary heart disease and stroke is not yet fully understood. In some randomized studies 
is reported that reduced levels of Hcy do not reduce the overall risk of cardiovascular disease (CVD) [18, 19].

Genest and colleagues (2000) noted the link between high levels of Hcy and atherosclerosis. The reliability of information derived mainly from case-control studies, however, the strength of this association is not reliable in prospective studies. Thus the causal link between total Hcy and heart disease is not so reliable as to make recommendations regarding screening and treatment for the prevention of CVD [20].

American Academy of Family Physicians (AAFP 2012) concluded that for coronary heart disease, the current evidence is insufficient to assess the balance of benefits and harm of using non-traditional risk factors for prevention of vascular events.

Non-traditional risk factors included in this recommendation are highly sensitive C-reactive protein, ankle-brachial index, leukocyte count, fasting levels of blood sugar, gum disease, the thickness of the carotid intima, coronary artery calcification, HCY levels and lipoprotein (a) level and other.

In patients with end-stage renal damage that have much greater degree for risk of vascular events or death from such an incident is extremely important to determine to what extent these factors-traditional and non-traditional influence.

Therefore, in our research we studied 120 hemodialysis patients and have them monitored for cardiovascular events (heart attack or stroke) for one year.

\section{Materials and Methods}

In our study, we studied 120 hemodialysis patients, women and men aged from 18 to 80 years, in a V stage CKD. Except hemodialysis patients are examined and healthy subjects as controls without renal or cardiovascular disease. All studied patients have consented to their results to be used for scientific purposes.
Patients were followed for one year within six visits for development of cardiovascular event (heart attack or stroke) or death due to such. In 18 patients (10 women and 8 men) has come vascular events-heart attack or stroke, compared with 15 of them whose outcome was fatal. All samples were analyzed with biochemical analyzer Cobas Integra 400 in medical diagnostic laboratory of University Hospital "Dr. George Stransky"-Pleven.

The biological material is serum, without hemolysis and lipemia.

Patients in the course of a year are monitored for homocysteine, albumin, cholesterol, triglycerides, LDL-cholesterol, and creatinine.

We use Roche assay tests, based on routinely principles to determine a laboratory parameters used in the clinical laboratory. Determination of homocysteine is the principle of testing the enzymatic cycle and evaluates the product from the conversion of cosubstrate instead evaluating itself cosubstrate or products from the conversion of homocysteine. The concentration of homocysteine in the sample is proportional to the amount of NADH, NAD + in turn. For the determination of serum creatinine is used an enzyme method, not the widely used test based on the principle of Jaffe. The reference values are accepted for the laboratory parameters of healthy individuals of the Bulgarian population are: albumin $35-50 \mathrm{~g} / \mathrm{L}$, Hcy $<12 \mathrm{umol} / \mathrm{L}, \mathrm{Tg}<2.3 \mathrm{mmol} / \mathrm{L}, \mathrm{Chol}<5.2 \mathrm{mmol} / \mathrm{L}$, LDL cholesterol $<3.36 \mathrm{mmol} / \mathrm{L}$, CreaP women $<84$ umol/L, male $<104 \mathrm{umol} / \mathrm{L}$.

Using a statistical program is done a research on the quantitative characteristics and prognostic capabilities of homocysteine and other biochemical parameters. We determined the diagnostic specificity and sensitivity of our lab performance against vascular disease (heart attack or stroke) by ROC curves.

\section{Results and Discussion}

The diagnostic quality of the researched by us laboratory indicators is evaluated by ROC curves 
based on the sensitivity and specificity of the tests. (Table. 1)

Under Table 1 Sensitivity is defined by the proportion of positive cases of the test (s) in the actually sick $(a+b)$ to the group itself, i.e. attitude and/ $(a+b)$, and specificity - the attitude of negative test cases according to $(d)$ in the healthy group $(c+d)$, i.e. to the relation $\mathrm{d} /(\mathrm{c}+\mathrm{d})$.

For each of the observed values of biochemical parameters we calculated the sensitivity and specificity. The threshold values for which the parameters have the highest sensitivity and specificity are shown in Table 2

The pairs of the corresponding numbers for their sensitivity and specificity of a given indicator are reflected as points on the rectangular coordinate system. Once joined, the resulting polyline is precisely the ROC curve (Fig. 1). The area below is a measure of to what extent the values of biochemical indicator patients in the sample can be classified into one of two groups-patients/healthy.

Fig. 1 shows the ROC curves of the 6 biochemical parameters of important diagnostic role in cardiovascular disease, namely curves: albumin (a), cholesterol (b), LDL (c), creatinine (g), homocysteine (e), TG (f).

Summary of diagnostic value of parameters to judge the coefficient AUC - area under the curve
(Table 3). For cholesterol, LDL, triglycerides, albumin, and it was a significant $(P<0.05)$ and exceeds 0.66 , i.e..

Each of these laboratory parameters can properly diagnose sick and healthy people over $66 \%$ of patients in the sample $(\mathrm{N}=120)$ compared to cardiovascular disease. For the entire population of patients with chronic kidney disease on hemodialysis (V stage) this percentage ranged from $52.3 \%-80.4 \%$ (TG) to 62.0\%-89.3\% (albumin).

With diagnostic value are creatinine and homocysteine. Their $P$-values $(0.087$ and 0.089 respectively) are greater than the critical point of 0.05 but less than 0.10 - the highest significance level, which also applies (albeit less frequently) in biostatistical research. Under this condition, homocysteine may also be used as a diagnostic test against cardiovascular disease. For Hcy AUC $=0.632$ $95 \% \mathrm{CI}=0.476 \div 0.788$. Its threshold values are $>$ 47.30 that determine sensitivity of $62.5 \%$ and specificity $55.8 \%$ (Table 2).

However, the diagnostic value of homocysteine is particularly high in terms of two categories - patients on dialysis and controls. ROC curve for this case is represented in Fig. 2.

AUC ratio is equal to 0.994 (p0000) with $95 \% \mathrm{CI}=$ $0.985 \div 1.000$. Homocysteine is practically 100 percent of properly diagnosed group of 140 persons of two

Table 1 Evaluation of diagnostic tests.

\begin{tabular}{llll}
\hline Diagnosis & & Testresults & All \\
\hline & Positive & Negative & \\
Sick & $\mathrm{a}$ & $\mathrm{b}$ & $\mathrm{a}+\mathrm{b}$ \\
Healthy & $\mathrm{c}$ & $\mathrm{d}$ & $\mathrm{c}+\mathrm{d}$ \\
All & $\mathrm{a}+\mathrm{c}$ & $\mathrm{b}+\mathrm{d}$ & $\mathrm{N}$ \\
\hline
\end{tabular}

Table 2 The threshold values of biochemical parameters.

\begin{tabular}{llll}
\hline Parameter & Thresholdvalue & Sensitivity $\%$ & Specificity\% \\
\hline Albumin & $\leq 37.2$ & 69.2 & 68.8 \\
LDL & $\leq 2.08$ & 66.3 & 68.8 \\
Cholesterol & $\leq 3.97$ & 65.4 & 62.5 \\
Triglycerides & $\leq 1.40$ & 62.5 & 62.5 \\
Creatinine & $\leq 720.00$ & 64.4 & 66.3 \\
Homocysteine & $\geq 47.30$ & 62.5 & 55.8 \\
\hline
\end{tabular}


The Role of Homocysteine and Other Clinical Laboratory Markers in Assessing

Cardiovascular Risk in Patients on Hemodialysis
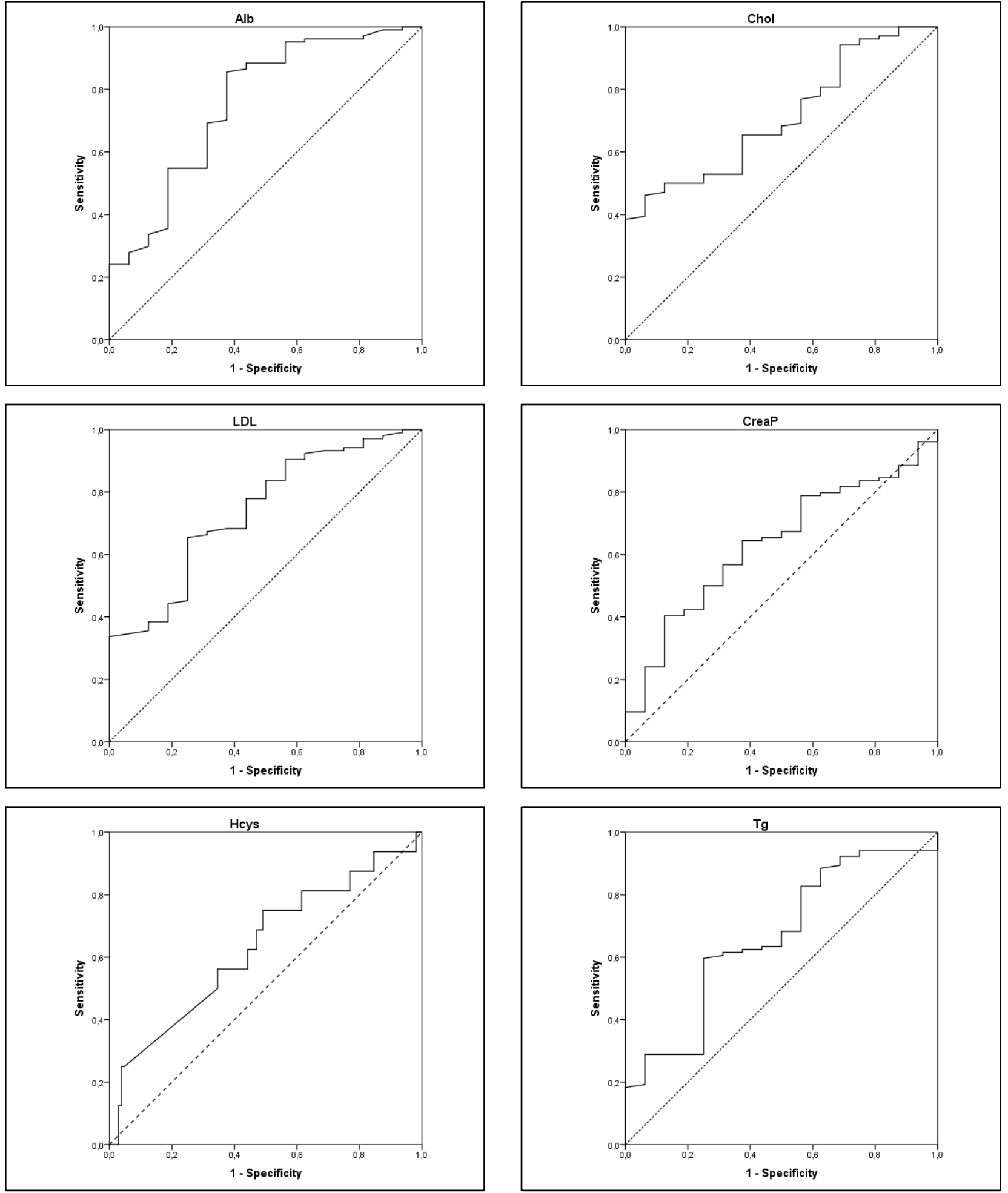

Fig. 1 ROC curves biochemical parameters against cardiovascular disease. 
Table 3 AUC - coefficients against cardiovascular disease.

\begin{tabular}{llllll}
\hline Parameter & AUC & SE & $P$ & & $95 \%$ CI \\
\hline Tg & 0.664 & 0.072 & 0.035 & 0.523 & 0.804 \\
Chol & 0.710 & 0.060 & 0.007 & 0.593 & 0.828 \\
LDL & 0.739 & 0.064 & 0.002 & 0.614 & 0.864 \\
Alb & 0.757 & 0.070 & 0.001 & 0.620 & 0.893 \\
CreaP & 0.633 & 0.067 & 0.087 & 0.501 & 0.766 \\
Hcys & 0.632 & 0.080 & 0.089 & 0.476 & 0.788 \\
\hline
\end{tabular}

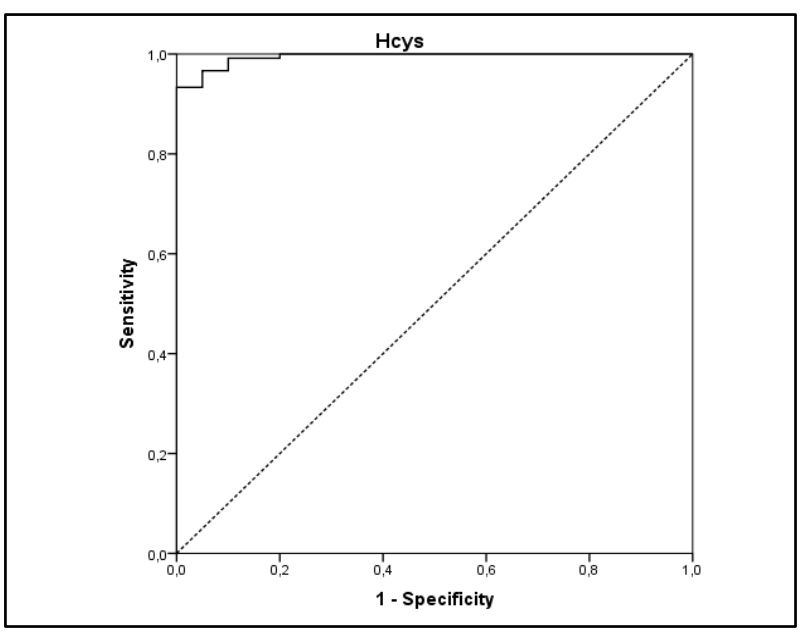

Fig. 2 ROC curve of homocysteine in dialysis patientst and controls.

categories: 120 - patients with chronic renal damage and 20-healthy controls without kidney disease. There is a full coverage with reality. The threshold values are $\geq 15.30$, which defines sensitivity of $99.2 \%$ and specificity $90.0 \%$. These results support the thesis of the diagnostic value of homocysteine in certain disease manifestations in patients on hemodialysis.

\section{Conclusions}

In conclusion we can say that there is a relation between hyperhomocysteinemia and cardiovascular events in patients on dialysis. Homocysteine and the rest of the studied by us laboratory parameters can be regarded as laboratory markers of choice for assessing the risk of heart attack or stroke in patients on dialysis. The six parameters are high diagnostic sensitivity and specificity of the risk of vascular events in patients with end-stage renal damage.

\section{References}

[1] Raine, A. E., Margreiter, R., and Brunner, F. P., et al.
1992. "Report on Management of Renal Failure in Europe XXII.” 1991 Nephrol. Dial. Transplant. 7 (2): 7-35.

[2] Levey, A. S. 1998. "Controlling the Epidemic of Cardiovascular Disease in Chronic Renal Disease: Where do We Start?" Am. J. Kidney Dis. 32 (3): 5-13. Wilcken, D. E., and Gupta, V. J. 1979. "Sulphr Containing Amino Acids in Chronic Renal Failure with Particular Reference to Homocystine and Cysteine-homocysteine Mixed Disulphide." Eur. J. Clin. Invest 9: 301-7.

[3] Chauveau, P., Chadefaux, B., and Coude, M., et al. 1993. "Hyperhomocysteinemia, a Risk Factor for Atherosclerosis in Chronic Uremic Patients." Kidney Int. 43 (41): 72-7.

[4] Bachmann, J., Tepel, M., and Raidt, H., et al. 1995. "Hyperhomocysteinemia and the Risk for Vascular Disease in Hemodialysis Patients." J. Am. Soc. Nephrol.: 121-5.

[5] Bostom, A. G., Shemin, D., and Lapane, K. L., et al. 1995. "Hyperhomocysteinemia and Traditional Cardiovascular Disease Risk Factors in End-stage Renal Disease Patients on Dialysis: A Case-control Study." Atherosclerosis 114: 93-1037. Hultberg, B., Andersson, A., and Sterner, G. 1993. "Plasma Homocysteine in Renal Failure." Clin. Nephrol. 41: 230-4.

[6] Robinson, K., Gupta, A., and Dennis, V., et al. 1996. "Hyperhomocysteinemia Confers an Independent Increased Risk of Atherosclerosis in End-stage Renal Disease and is Closely Linked to Plasma Folate and Pyridoxine Concentrations." Circulation 94: 2743-8.

[7] Bostom, A., Brosnan, J. T., and Hall, B., et al. 1995. "Net Uptake of Plasma Homocysteine by the Rat Kidney in vivo." Atherosclerosis 116: 59-62.

[8] Guttormsen, A. B., Svarstad, E., Ueland, P. M., and Refsum, H. 1995. "Elimination of Homocysteine from Plasma in Subjects with End Stage Renal Failure." Ir. J. Med. Sci. 164 (15): 8-9.

[9] Vychytil, A., Fodinger, M., and Papagiannopoulos, M., et al. 1999. "Peritoneal Elimination of Homocysteine Moieties in Continuous Ambulatory Peritoneal Dialysis Patients." Kidney Int. 55: 2054-61 http://dx.doi.org/10.1046/j.1523-1755.1999.00437.

[10] Sirrs, S., Duncan, L., and Djurdjev, O., et al. 1999. 


\section{Cardiovascular Risk in Patients on Hemodialysis}

"Homocysteine and Vascular Access Complications in Haemodialysis Patients: Insights into a Complex Metabolic Relationship." Nephrol. Dial. Transplant. 14: 738-43.

[11] Blacher, J., Demuth, K., and Guerin, A. P., et al. 1998. "Influence of Biochemical Alterations on Arterial Stiffness in Patients with End Stage Renal Disease." Arterioscler. Thromb. Vasc. Biol. 18: 534-41.

[12] Blacher, J., Demuth, K., and Guerin, A. P., et al. 1999. "Association between Plasma Homocysteine Concentrations and Cardiac Hypertrophy in End Stage Renal Disease." J. Nephrol. 12: 248-55.

[13] Bostom, A. G., Shemin, D., and Verhoef, P., et al. 1997. "Elevated Fasting Total Plasma Homocysteine Levels and Cardiovascular Disease Outcomes in Maintenance Dialysis Patients." Arterioscler. Thromb. Vasc. Biol. 17: 2554-8.

[14] Moustapha, A., Naso, A., and Nahlawi, M., et al. 1998. "Prospective Study of Hyperhomocysteinemia as an Adverse Cardiovascular Risk Factor in End-stage Renal Disease." Circulation 97: 138-41.

[15] Suliman, M. E., Qureshi, A. R., and Barany, P., et al.
2000. "Hyperhomocysteinemia, Nutritional Status, and Cardiovascular Disease in Hemodialysis Patients." Kidney Int. 57: 1727-35 http://dx.doi.org/10.1046/j.1523-1755.2000.00018.x

[16] Foley, R. N., Parfrey, P. S., and Harnett, J. D., et al. 1996. "Hypoalbuminemia, Cardiac Morbidity and Mortality in End-stage Renal Disease." J. Am. Soc. Nephrol. 7: 728-36.

[17] Zoccali, C., Mallamaci, F., and Tripepi, G., et al. 1999. "Prediction of Left Ventricular Geometry by Clinic, Pre-dialysis and 24-h Ambulatory BP Monitoring in Hemodialysis Patients." J. Hypertens 17: 1751-8.

[18] Genest, J., McPherson, R., Frohlich, J., Anderson, T., Campbell, N., Carpentier, A., Couture, P., Dufour, R., Fodor, G., Francis, G. A., Grover, S., Gupta, M., Hegele, R. A., Lau, D. C., Leiter, L., Lewis, G. F., Lonn, E., Mancini, G. B., Ng, D., Pearson, G. J., Sniderman, A., Stone, J. A., and Ur, E. 2009. Canadian Cardiovascular Society/Canadian Guidelines for the Diagnosis and Treatment of Dyslipidemia and Prevention of Cardiovascular Disease in the Adult-2009 Recommendations. 